\title{
Photostimulated etching of germanium chalcogenide films
}

\author{
V.A. Dan'ko, I.Z. Indutnyi, V.I. Myn'ko, P.E. Shepeliavyi, M.V. Lukyanyuk, O.S. Litvin \\ V. Lashkaryov Institute of Semiconductor Physics, National Academy of Sciences of Ukraine, \\ 41, prospect Nauky,03028Kyiv, Ukraine,e-mail: indutnyy@isp.kiev.ua
}

\begin{abstract}
The new effect of photostimulated dissolution of as-evaporated and annealed Ge-based chalcogenide glass $(\mathrm{ChG})$ films was investigated in detail. The etching rate increases with the illumination intensity, and its spectral dependence is correlated with absorption in the film at the absorption edge. A possible mechanism for the photoinduced etching of $\mathrm{ChG}$ films has been discussed. The high-frequency diffraction gratings on germanium $\mathrm{ChG}$ - more environmentally acceptable compounds than traditionally used arsenic chalcogenides - were recorded using the method of interference immersion photolithography with photoinduced etching.
\end{abstract}

Keywords: chalcogenide glasses, photoinduced dissolution.

Manuscript received 07.08.12; revised version received 14.09.12; accepted for publication 17.10.12; published online 12.12.12.

\section{Introduction}

Chalcogenide vitreous semiconductors, or, in other words, chalcogenide glasses (ChG), attract attention of many researchers owing to widely diverse photoinduced changes in their structure and, as a consequence, in their properties (optical characteristics, conductivity, solubility in selective etchants, and even mechanical characteristics). These modifications serve as a basis for the practical use of $\mathrm{ChG}$ as inorganic photoresists [1-4], media for optical and electrical information recording [5-7], and other applications [8,9].

Photoinduced modifications of $\mathrm{ChG}$ films deposited using thermal evaporation in vacuum have two components: reversible and irreversible $[5,6]$. The use of chalcogenide films as photoresists is specifically related with irreversible changes in their solubility. ChG films thermally deposited in vacuum have a considerable amount of "anomalous" homopolar bonds. Pores and voids are also present, even if these films have stoichiometric composition. Illumination or annealing causes polymerization of molecular groups into the basic matrix of chalcogenide glass, which is accompanied by decreasing the number of homopolar bonds and voids, and it is this circumstance that changes the physical-andchemical properties of ChG films and, in particular, their solubility. Sufficiently high etching selectivity is observed in this process only in arsenic-based chalcogenide films, such as As-S, As-Se and As-S-Se. ChG films based on germanium are low-sensitive and deposition of an additional silver layer is necessary for their use as a photoresist, which markedly complicates the technological process. Moreover, annealed ChG films, in which thermally stimulated polymerization of the structure has already occurred, and films deposited by a non-thermal method (laser or magnetron sputtering in vacuum, deposition from solutions) are also insensitive (characterized by low etching selectivity).

Photostimulated effects in ChG have been mostly studied in films that are freshly deposited, annealed, and illuminated, with analysis of changes in the structure and physical-and-chemical properties of a film as a result of exposure to light. However, investigations performed in situ under exposure to light have shown $[10,11]$ that transient photoinduced structural changes are also observed in ChG films. These changes are only observed during illumination of the films and rapidly relax after switching off the light.

In our previous investigations, it was shown for the first time that these transient structure changes in $\mathrm{ChG}$ films (including the annealed ones) are accompanied by a change in the solubility of chalcogenide in selective etchants, and negative amine-based etchants dissolve illuminated areas of chalcogenide films, i.e. act as positive etchants $[12,13]$. This new photostimulated effect allows to realize the photolithographic process on annealed ChG layers (including Ge-based films) and deposited using non-thermal methods with simultaneous exposure and selective etching of these layers. In this paper, we report on the results of investigations of this 


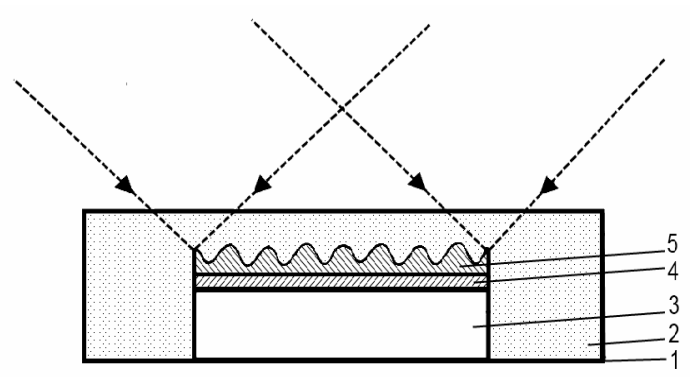

a

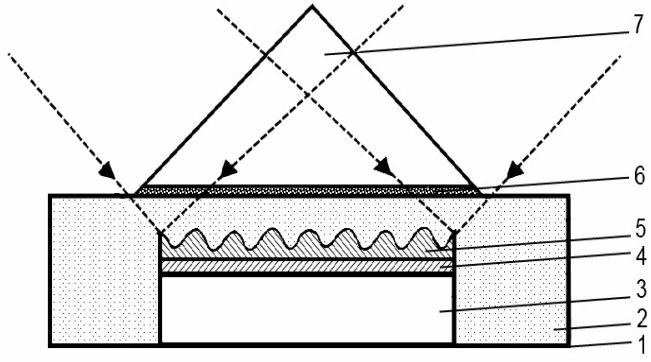

b

Fig. 1. The scheme of photoinduced etching for $\mathrm{ChG}$ film in interference pattern of coherent laser radiation $(a)$ and the same with using the prism $(b)$ : 1 - silica cuvette filled with selective etching solutions (2), 3 - substrate, 4 - adhesive layer of $\mathrm{Cr}, 5$ chalcogenide photoresist, 6 -immersion substance, 7 - prism.

effect in Ge-based chalcogenide films and realization of interference photolithography (including immersion interference lithography) in these layers.

\section{Experiment}

The samples were prepared by successive thermal evaporation in vacuum at a residual pressure of $2 \cdot 10^{-3} \mathrm{~Pa}$, with a 6-nm thick (effective thickness) adhesive layer of $\mathrm{Cr}$ and $\mathrm{ChG}$ layers with thicknesses from 200 up to $600 \mathrm{~nm}$, deposited onto substrates. Polished glass and silicon (orientation $\{111\}$ ) plates are used as substrates. The layer thickness was controlled during deposition with a KIT-1 quartz micro-balance and measured by MII-4 microinterferometer upon deposition. Some of deposited films were annealed for 0.5 to $2 \mathrm{~h}$ at temperatures 5 to $15^{\circ} \mathrm{C}$ lower than $T_{g}$, where $T_{g}$ is the glass-transition temperature of a given chalcogenide.

The etching rates of ChG films were studied using the quartz-oscillator method [14] in a silica cuvette filled with a selective etchant [15] based on amine solutions. The exposure of the samples during etching was performed using the integral light of a DRSh-250 mercury lamp. Spectral emission lines of the same lamp were selected by filters for investigation of spectral dependences for the photoetching rate.

Interference structures on the $\mathrm{ChG}$ films were recorded using their exposure to an interference pattern [16] formed by the light of a helium-cadmium laser (wavelength $\lambda=440 \mathrm{~nm}$ ) with the holographic setup assembled in accord with the wave-amplitude division method. During exposure, these samples were also placed into the silica cuvette filled with selective etching solutions (Fig. 1a). For decreasing the grating period, a glass prism was used (Fig. 1b). In this method of immersion interference lithography, the prism was applied to the silica cuvette using immersion liquid. This method allows to increase the spatial frequency of a grating by $n$ times, where $n$ is the refraction index of the prism material.
The profile shape of the obtained structures was investigated using a Dimension 3000 Scanning Probe atomic force microscope (AFM) (Digital Instruments Inc.). The diffraction properties of the obtained relief gratings were studied by measuring the spectral dependence of the diffraction efficiency $\eta(\lambda)$ of samples preliminarily coated with a 40 -nm-thick Al reflecting layer. Value $\eta$ was taken as the ratio of the diffracted beam intensity to that of the incident beam, and were measured for $s$ - and $p$-polarized light (electric vector of light-wave is perpendicular and parallel to grating grooves, respectively). Spectral measurements were performed for the first order of diffraction by using the setup close to the Littrow scheme, the angle between the incident and diffracted beams was close to $8 \mathrm{deg}$. The angular dependence $\eta(\varphi)$ was measured using a heliumneon laser $(\lambda=632.8 \mathrm{~nm})$.

\section{Results and discussion}

Fig. 2 shows the kinetic curves $d(t)$ for the etching of $\mathrm{Ge}_{25} \mathrm{Se}_{75}$ layers in a selective amine-based etchant [15]. The initial thickness of these layers was $d_{0}=300 \mathrm{~nm}(d$ is the residual layer thickness after etching, and $t$ is the duration of etching): 1 - as-deposited unexposed layer, 2 - annealed unexposed layer, 3- as-deposited layer illuminated during etching by integral radiation of a mercury lamp $(250 \mathrm{~W})$ with the energy density of incident radiation $\mathrm{p}=8.8 \mathrm{~mW} / \mathrm{cm}^{2}, 4-$ annealed layer illuminated by the same radiation during etching.

It can be seen in Fig. 2 that selected non-water etchant based on amine solution is characterized by good selectivity for the annealed $\mathrm{Ge}_{25} \mathrm{Se}_{75}$ layer (value of selectivity defined by the ratio of the dissolution rates) for exposed and unexposed layers reached 20). But in contrast to traditional lithography on $\mathrm{ChG}$ photoresists which use thermally deposited (and non-annealed) ChG layers and where there is a negative selectivity (unexposed photoresist areas are dissolved faster, than exposed), in the photoetching case those layers dissolve faster that are illuminated in the process of etching (curves 3 and 4, as compared with the curves 1 and 2). 


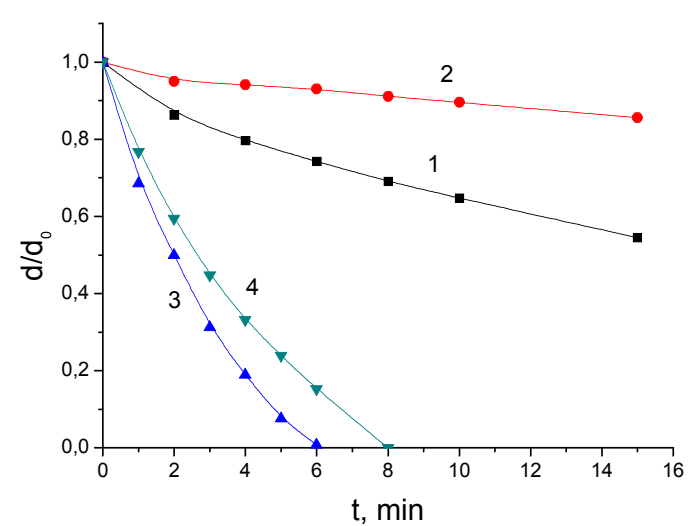

Fig. 2. Dissolution kinetics of $\mathrm{Ge}_{25} \mathrm{Se}_{75}$ layers in the aminebased etchant: 1 - as-deposited unexposed layer, 2 - annealed and unexposed layer, 3 - as-deposited layer exposed during etching by integral radiation of the mercury lamp $(250 \mathrm{~W})$ with the energy density of incident radiation $p=8.8 \mathrm{~mW} / \mathrm{cm}^{2}, 4-$ annealed layer exposed during etching by the same lamp.

The dissolution rate increases with increasing the radiation intensity non-linearly: six-fold increase in light power results in three times reduction in the time of complete film dissolution. For germanium chalcogenides, the photoetching effect occurs both on annealed and as-evaporated layers, but selectivity of etching is slightly high on the annealed layers.

The spectral dependence of the photoinduced etching efficiency for $\mathrm{ChG}$ layers correlates well with the spectral dependence of the absorption index $k$ near the absorption edge of these films [17]. However, within the short-wave spectral range the photoetching efficiency slightly decreases. This decrease in efficiency may be caused by the absorption of short-wave radiation in the etchant solution.

Similar results with certain quantitative details were obtained for arsenic chalcogenide films, but for the arsenic-based ChG the photoetching effect occurs only in annealed layers [12].

What is the mechanism of the fast dissolution of ChG films (including the annealed ones and deposited by non-thermal methods) during the course of its illumination, i.e., why does the photoinduced etching effect appear? It may be assumed that, at a sufficient illumination intensity at which a substantial concentration of photoexcited carriers is generated in a film, the structure of the film somehow changes. This suggestion was confirmed by in situ EXAFS, ESR and Raman investigations of reversible photostructural changes in ChG $[10,18,19]$. It has been shown that the initial step of the process is formation of dynamic interchain bonds through photoinduced unpairing of lone-pair electrons. Subsequent bond relaxation and rupture result in a metastable structural change.

Transient changes in the $\mathrm{ChG}$ structure during illumination are also confirmed by the extensively studied phenomenon of photostimulated flow (photoinduced plasticity) in films of binary chalcogenides and selenium [20,21]. This phenomenon is characterized by a decrease in viscosity and an increase in fluidity of $\mathrm{ChG}$ films exposed to light with sufficient intensity and is only observed during illumination of ChG. Several microscopic mechanisms of photoinduced plasticity have been suggested [18, 22]. Fritzsche [22] attributed photoinduced plasticity to the light-stimulated generation of pairs with variable valence and to the recombination-stimulated displacement of atoms. The recombination-induced bond rearrangements continue as long as $\mathrm{ChG}$ is exposed to electron-hole producing light, and this dynamic state is comparable to the structural state near the glass-transition temperature. Other authors [18], being based on in situ EXAFS and Raman experiments, propose two possible mechanisms, namely: (i) an interchange of covalent bonds through bond rupture and rebonding (which is supported by EXAFS results [23]) and (ii) weakening the intermolecular and interchain bonds (which agree with Raman measurements [24]).

These transient structure changes, as shown by our investigations [12], are accompanied by a change in solubility of chalcogenides in selective etchants. It can be assumed that photodissolution of $\mathrm{ChG}$ is probably stimulated just by bond rearrangements during their exposure to photoactive light.

The results obtained by us enable to use the photoinduced etching of ChGs in formation of lithographic masks or profiled interference structures [12]. Photolithography based on this effect has a number of advantages in comparison with conventional use of ChG films as photoresists. It can be realized on annealed chalcogenide layers, which are characterized by a lower defect concentration than that in thermally as-deposited films. This makes it possible to obtain higher quality lithographic masks or periodic profiled phase structures.

Another advantage of photolithography using the photoetching effect is that the processes of etching and exposure in this method are combined, which diminishes the number of technological operations in the photolithographic process. In photoetching lithography for deposition of chalcogenide films, more technological methods can be also used: electron-beam evaporation, laser or high-frequency magnetron sputtering, deposition on the substrate from solutions. Being annealed at a temperature close to the glass-transition point, all chalcogenide films have properties close to those of a chalcogenide glass of corresponding composition, irrespective of the deposition method.

This effect has been used to fabricate diffraction gratings on germanium $\mathrm{ChG}$ - more environmentally acceptable compounds than traditionally used arsenic chalcogenides.

Fig. 3 shows AFM picture of the diffraction grating that was formed on the annealed $\mathrm{Ge}_{25} \mathrm{Se}_{75}$ film (the initial thickness of this layer was $d_{0}=200 \mathrm{~nm}$ ) by photoetching method with using the same amine-based etchant. The spatial frequency of grooves in the recorded 

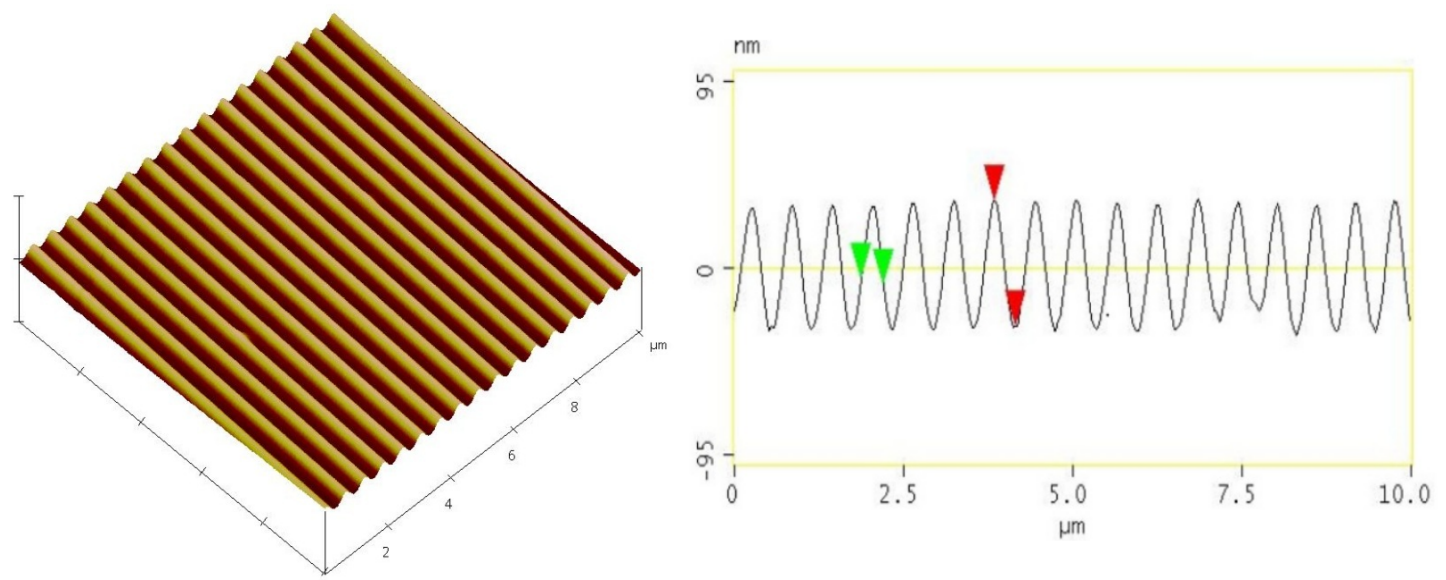

Fig. 3. AFM image of the diffraction grating recorded in a $\mathrm{Ge}_{25} \mathrm{Se}_{75}$ layer by using the method of interference lithography with photoinduced etching and the profile of its grooves.
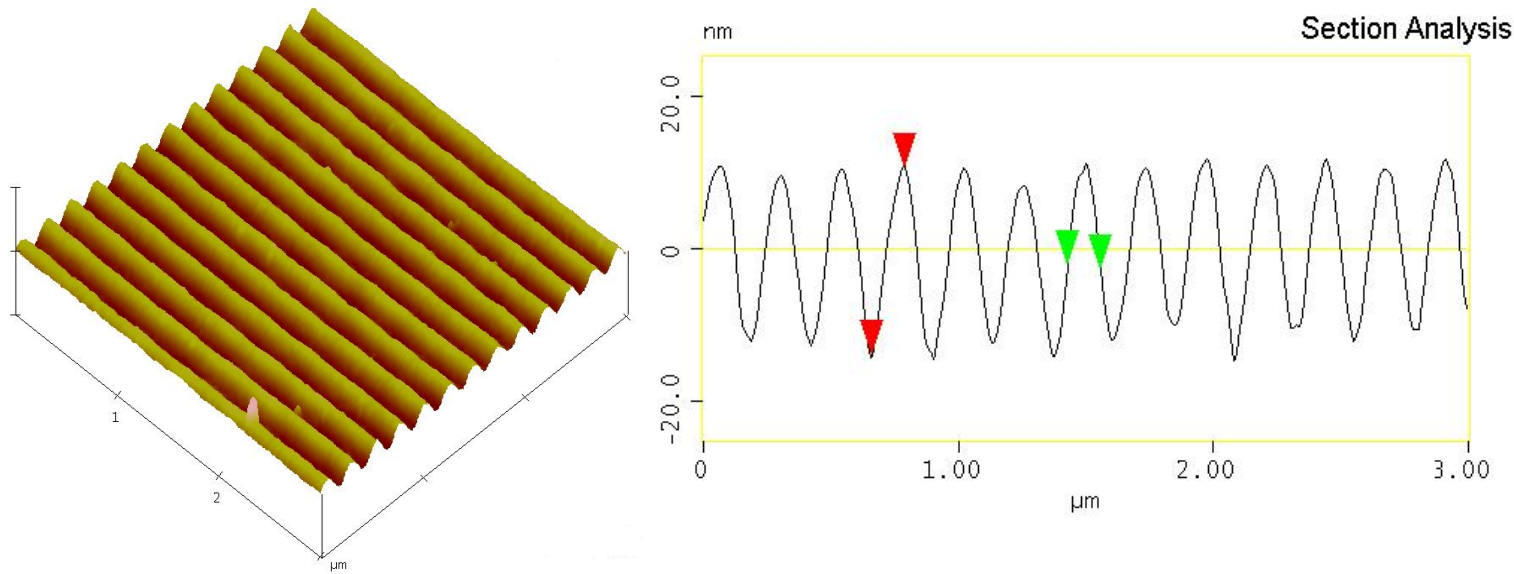

Fig. 4. AFM image of the diffraction grating recorded in an $\mathrm{Ge}_{25} \mathrm{Se}_{75}$ layer by using the method of immersion interference lithography with photoinduced etching and the profile of its grooves.

grating is $1700 \mathrm{~mm}^{-1}$; the profile depth is $65 \mathrm{~nm}$. The shape of the groove profile of the grating is nearly sinusoidal. The recording time of the grating is $20 \mathrm{~min}$, and its size is $30 \times 30 \mathrm{~mm}$ at a laser power of $25 \mathrm{~mW}$. During exposure to the absorbed radiation of a heliumcadmium laser (wavelength $\lambda=440 \mathrm{~nm}$ ), the diffraction efficiency of the formed grating was monitored in situ with the non-photoactive light of a helium-neon laser $(\lambda=632.8 \mathrm{~nm})$. The increase in photoetching time (or power of laser) allows to obtain gratings with a higher relief depth.

To increase in the spatial frequency of gratings, we used a glass prism with $n=1.5$, which was applied to a silica cuvette using immersion liquid (Fig. 1). Fig. 4 shows AFM image of the obtained diffraction grating formed on the $\mathrm{Ge}_{25} \mathrm{Se}_{75}$ film by this method of immersion interference lithography with photoinduced etching. The spatial frequency of grooves in the recorded grating was $4330 \mathrm{~mm}^{-1}$; the profile depth $-25 \mathrm{~nm}$. The shape of the groove profile of the grating was nearly sinusoidal, too. The recording time (when using a helium-cadmium laser, $\lambda=440 \mathrm{~nm}$ ) for the grating was $50 \mathrm{~min}$, and its size was $30 \times 30 \mathrm{~mm}$ for the laser power $25 \mathrm{~mW}$.

The diffraction structures formed in this way have corresponding optical properties of holographic diffraction gratings. Fig. 5 shows the spectral dependences of diffraction efficiency for a grating (with the profile shown in Fig. 3) coated with a reflective $\mathrm{Al}$ layer (thickness $40 \mathrm{~nm}$ ). The curves 1 and 2 correspond to perpendicular and parallel orientation of the light wave electric vector relatively to the grating grooves.

Fig. 6 shows the dependence of the diffraction efficiency on the angle of light incidence $\eta(\varphi)$ for the sample shown in Fig. 3. Results for both polarizations were obtained.

Relations between diffraction characteristics of a grating and their groove profile as well as the depth of modulation were investigated by many authors [25-27]. 


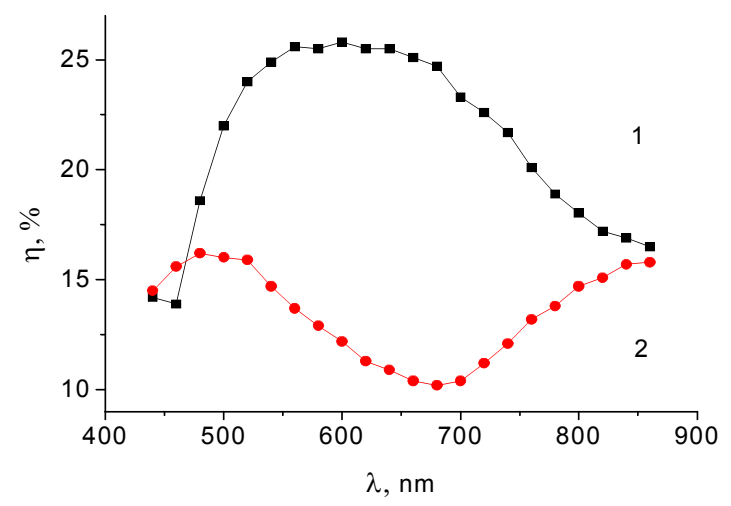

Fig. 5. Spectral dependence of the diffraction efficiency $\eta$ for the grating formed by photoetching (the same sample as in Fig. 3): curves 1 and 2 correspond, respectively, to perpendicular and parallel orientation of the light wave electric vector relatively to the grating grooves.

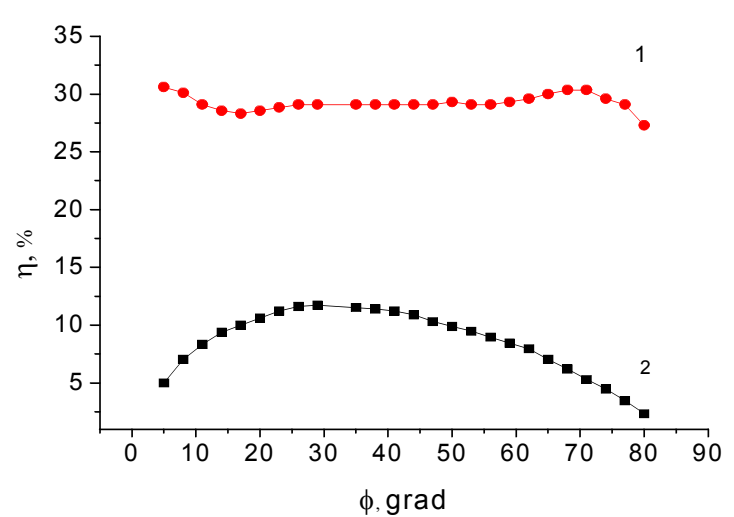

Fig. 6. Angular dependence of the diffraction efficiency $\eta$ for the grating formed by photoetching (the same sample as in Fig. 3): curves 1 and 2 correspond, respectively, to perpendicular and parallel orientation of the light wave electric vector relatively to the grating grooves.

In particular, in [27] the spectral dependence of the diffraction efficiency of symmetric gratings with various forms of the grooves was investigated. The authors showed that, by changing the groove profile, the maximum $\eta$ position on the wavelength axis and width of the operation interval of the grating can be controlled. If we compare our results in Figs 5 and 6 with theoretically calculated characteristics for gratings with sinusoidal groove profile, we can note qualitative agreement between the angular and spectral $\eta$ dependences. As can be seen from the analysis of AFM images (Figs 3 and 4), profiles of our gratings are close to the sinusoidal one, as it is postulated in the theoretical calculations. These results are consistent with the quasilinear kinetics of $\mathrm{ChG}$ photoetching (Fig. 2), at the initial part of the dissolution kinetics, for small changes in thickness of ChG layer (in our case $d / d_{0} \sim 0.7-0.8$ ).

\section{Conclusions}

The simultaneous illumination and etching of asevaporated and annealed Ge-based ChG films results in the photoinduced enhancement in the solubility of the chalcogenides in selective etchants. It was shown that negative-action etchants based on amines dissolve illuminated portions of a chalcogenide film, i.e., act as positive etchants. It can be assumed that the photodissolution of $\mathrm{ChGs}$ is stimulated by bond rearrangements under exposure to a photoactive light.

The effect of photostimulated dissolution has been used for fabrication of diffraction gratings in germanium ChG films by interference immersion photolithography. Relief parameters and diffraction properties of the obtained structures have been studied. The photoetching technique used to form interference periodic structures onto chalcogenide layers is simple, inexpensive, and adaptable to large-scale manufacturing.

\section{References}

1. Y. Mizushima and A. Yoshikava, Photoprocessing and lithographic applications, in: Amorphous Semiconductors Technologies and Devices (Y. Hamakava, Ed.). OHM, Tokyo, and NorthHolland, Amsterdam, 1982, p. 277-295.

2. G.H. Bernstein, W.P. Liu, Y.N. Khawaja, M.N. Kozicki and D.K. Ferry, High resolution electron beam lithography with negative and inorganic resists // J. Vac. Sci. Technol. B6(6), p. 2298-2307 (1988).

3. K. Saito, Y. Utsugi, and A. Yoshikawa, X-ray lithography with $\mathrm{Ag}-\mathrm{Se} / \mathrm{Ge}-\mathrm{Se}$ inorganic resist using synchrotron radiation // J. Appl. Phys. 63(2), p. 565-567 (1988).

4. S.H. Wong, M. Thiel, P. Brodersen, D. Fenske, G.A. Ozin, M. Wegener, and G. von Freymann, Highly selective wet etch for high-resolution threedimensional nanostructures in arsenic sulfide allinorganic photoresist // Chem. Mater. 19(17), p. 4213-4221 (2007).

5. M. Wuttig \& N. Yamada, Phase-change materials for rewriteable data storage // Nature Materials, 6, p. 824-832 (2007).

6. I.Z. Indutnyy, M.T. Kostyshyn, I.P. Kasjarum, V.I. Min'ko, E.V. Michailovska, P.F. Romanenko, Photostimulated Interactions in MetalSemiconductor Structures. Naukova Dumka, Kiev, 1992 (in Russian).

7. Photo-Induced Metastability in Amorphous Semiconductors, ed. by A.V. Kolobov. Wiley, New York, 2003.

8. K. Richardson, L. Petit, N. Carlie, B. Zdyrko, I. Luzinov, J. Hu, A. Agarval, L. Kimerling, T. Anderson and M. Richardson, Progress on the fabrication of on-chip integrated chalcogenide glass (ChG)-based sensors // J. Nonlinear Opt. Phys. \& Materials, 19(1), p. 75-99 (2010). 
9. M.-L. Anne, J. Keirsse, V. Nazabal, K. Hyodo, S. Inoue, C. Boussard-Pledel, H. Lhermite, J. Charrier, K. Yanakata, O. Loreal, J.Le Person, F. Colas, C. Compère, and B. Bureau, Chalcogenide glass optical waveguides for infrared biosensing // Sensors, 9, p. 7398-7411 (2009).

10. A.V. Kolobov, K. Tanaka, Nanoscale mechanism of photo-induced metastability and reversible photodarkening in chalcogenide vitreous semiconductors // Fizika i tekhnika poluprovodnikov, 32(8), p. 899-904 (1998), in Russian.

11. A. Ganjoo, H. Jain, S. Khalid, Atomistic observation of photo-expansion and photo-contraction in chalcogenide films by in situ EXAFS // J. NonCrystalline Solids, 354, p. 2673-2678 (2008).

12. V.A. Dan'ko, I.Z. Indutnyi, V.I. Min'ko, P.E. Shepelyavyi, O.V. Berezn'ova, and O.S. Litvin, Photoinduced etching of thin films of chalcogenide glassy semiconductors // Semiconductors, 46(4), p. 504-508 (2012).

13. V.A. Dan'ko, I.Z. Indutnyi, V.I. Min'ko, P.E. Shepelyavyi, M.V. Lukyanyuk, and O.S. Litvin, Interference photolithography using photoetching effect in chalcogenide films // J. NonOxide Glasses, 3(2), p. 13-18 (2012).

14. K. Shtekhelmaher, in: Thin-film Microelectronics, ed. by L. Hollend. Mir, Moscow, 1968 (in Russian).

15. Patent of the Russian Federation № 2008285, IC5 C 03 C 15/00, 23/00 (1994). Solution for negative etching of chalcogenide glasses, I.Z. Indutnyi, S.A. Kostyukevich, P.E. Shepeliavyi // Otkrytiia, izobreteniia, № 4. Publ. 1994 (in Russian).

16. V.A. Dan'ko, I.Z. Indutnyi, V.I. Min'ko, and P.E. Shepelyavyi, Interference photolithography with the use of resists on the basis of chalcogenide glassy semiconductors // Optoelectronics, Instrumentation and Data Processing, 46(5), p. 483-490 (2010).

17. A.I. Stetsun, Extended Abstract of Candidate's Dissertation. Institute of Semiconductor Physics, National Academy of Sciences of Ukraine, Kyiv, 1994.
18. A.V. Kolobov and K. Tanaka, in: Handbook of Advanced Electronic and Photonic Materials and Devices, ed. by H.S. Nalwa. Academic, San Diego, $\mathbf{5}(2), 2001$.

19. A.V. Kolobov and J. Tominaga, Chalcogenide glasses in optical recording: Recent progress // $\mathrm{J}$. Optoelectr. and Adv. Mater., 4(3), p. 679-686 (2002).

20. M.L. Trunov, P.M. Lytvyn, P.M. Nagy, and O.M. Dyachyns'ka, Real-time atomic force microscopy imaging of photoinduced surface deformation in $\mathrm{As}_{\mathrm{x}} \mathrm{Se}_{100-\mathrm{x}}$ chalcogenide films // Appl. Phys. Lett. 96, 111908 (2010).

21. K. Tanaka, N. Kawakami, and A. Odajima, Photoinduced elastic changes in amorphous $\mathrm{As}_{2} \mathrm{~S}_{3}$ films // Jpn. J. Appl. Phys., Part 1, 20(12), p. L874L876 (1981).

22. H. Fritzsche, Photo-induced fluidity of chalcogenide glasses // Solid State Communs. 99(3), p. 153-155 (1996).

23. A.V. Kolobov, H. Oyanagi, K. Tanaka and Ke Tanaka, Structural study of amorphous selenium by in situ EXAFS: Observation of photoinduced bond alternation // Phys. Rev. B, 55, p. 726-734 (1997).

24. V.V. Poborchii, A.V. Kolobov, and K. Tanaka, Photomelting of selenium at low temperature // Appl. Phys. Lett. 74(2), p. 215 (1999).

25. D. Maystre, R. Petit, Essai de determination theorique $\mathrm{du}$ profil optimal d'un reseau holographique // Opt. Communs. 4(1), p. 25-28 (1971).

26. E.G. Loewen, M. Neviere, D. Maystre, Grating efficiency theory as it applies to blazed and holographic gratings // Appl. Opt. 16(10), p. 27112721 (1977).

27. L.A. Dmitrieva, I.V. Golubenko, G.M. Savitskii, Diffraction efficiency of holographic gratings of symmetric profiles // Optiko-mekhanich. promyshlennost', N 1, p. 4-6 (1985), in Russian. 